\title{
Use of rapid point-of-care diagnostic tests for the elimination of congenital syphilis: what is the evidence?
}

\author{
Ranmini Kularatne $e^{a, b *}$ \\ ${ }^{a}$ Centre for HIV \& STI, National Institute for Communicable Diseases, Johannesburg, South Africa \\ ${ }^{b}$ Department of Clinical Microbiology \& Infectious Diseases, University of the Witwatersrand, Johannesburg, South Africa \\ *Email: ranminik@nicd.ac.za
}

\begin{abstract}
Congenital syphilis constitutes a major preventable public health problem, that has been targeted for elimination by the World Health Organisation. Adverse pregnancy outcomes occur in upto $80 \%$ of untreated maternal syphilis. National impact targets for elimination include $95 \%$ syphilis testing and treatment coverage for pregnant women. Screening and treatment of maternal syphilis should ideally happen at the first ante-natal clinic visit. This may be facilitated by the use of rapid point-of-care tests (POCTs), especially for healthcare centres with limited laboratory access. There are several commercial syphilis POCTs, some of which also screen for HIV infection. These have different technical specifications, and their performance characteristics vary when capillary fingerprick whole blood is used for testing in a clinic setting. Syphilis POCT implementation in ante-natal care is affordable and rational in resource-constrained settings; however, managers and policy makers should be aware of the various programmatic issues that need to be addressed in the preimplementation phase and monitored over time.
\end{abstract}

Keywords: Congenital syphilis, syphilis screening in pregnant women, syphilis point-of-care tests

\section{Introduction}

Congenital syphilis is a preventable infection, and one that still constitutes a major public health burden. The World Health Organization (WHO) estimated that in 2012 nearly one million pregnant women were infected with syphilis globally, resulting in 350000 adverse pregnancy outcomes. ${ }^{1}$ A recent meta-analysis has revealed that these adverse pregnancy outcomes, including stillbirths, preterm deliveries, low birthweight neonates and infants with features of congenital syphilis, may be expected in up to $80 \%$ of untreated maternal syphilis. ${ }^{2}$ In 2007, the WHO stated that elimination of mother-to-child transmission (EMTCT) of syphilis is one of the most cost-effective public health interventions, and provided guidance for country-level action. ${ }^{3}$ The 2014 WHO global guidance document on the criteria for EMTCT of syphilis and HIV set a minimum national impact target of $\leq 50$ cases of congenital syphilis per 100000 livebirths, as well as process targets of $\geq$ $95 \%$ syphilis testing and treatment coverage for pregnant women. ${ }^{4}$ In pursuit of these goals, the use of rapid point-ofcare tests (POCTs) for syphilis has been considered to enable provision of screening and treatment for pregnant women at the first antenatal clinic visit. Such screening tests would have to meet specific performance criteria, as stipulated by the WHO. These WHO 'ASSURED' criteria for POCTs include the following: affordability; adequate sensitivity and specificity; ease-of-use; rapidity and robustness; equipment-free testing; and deliverability and acceptability to end-users. ${ }^{5}$ In order to prevent mother-to-child transmission, screening for syphilis in pregnancy should ideally be done before the end of the first trimester and at least one dose of benzathine penicillin administered at least 30 days before delivery. ${ }^{4}$

This review provides an overview of available syphilis POCTs; their performance characteristics in various field settings; implementation requirements; and rationale for use in publicsector antenatal clinics in South Africa.

\section{Laboratory-based syphilis serological assays}

Syphilis testing in diagnostic laboratories is done using plasma or serum specimens that are likely to have a sufficiently high concentration of detectable antibodies to either treponemal or non-treponemal antigens. Non-treponemal assays such as rapid plasma reagin (RPR) detect antibodies to non-specific lipid material such as cardiolipin that are released by treponemes and damaged host cells during active syphilis infection, whereas specific treponemal assays detect IgG and/ or IgM and IgA to highly immunogenic Treponema pallidum membrane lipoproteins such as Tp47, Tp17 and Tp15. They may be manual, e.g. Treponema pallidum haemagglutination assay (TPHA) or automated, e.g. Treponema pallidum chemiluminescent immunoassay (CLIA). Table 1 gives an overview of the characteristics of laboratory serological tests used for the diagnosis of syphilis.

\section{Rapid syphilis point-of-care tests}

All currently available syphilis POCTs are immunochromatographic lateral flow assays that capture antibody in patients' plasma, serum or whole-blood specimens. The antibodies detected may be specific treponemal and/or non-treponemal, depending on assay type, and a positive result is depicted as a line (or dot in the case of the Multiplo rapid TP/HIV antibody test) on the assay strip. Each assay has a built-in control line that must be visible for result validity. Table 2 shows available commercial assay types and test specifications, as stated by the manufacturers. The assays have different technical specifications, including time to reading of results. Deviations from the recommended testing protocol may result in false-negative or false-positive results.

\section{Performance characteristics of syphilis POCTs in comparison with standard laboratory assays}

\section{Field versus laboratory-based evaluations}

A systemic review and meta-analysis of 33 studies revealed that the sensitivity of POCT assays with whole blood was consistently 
Table 1: Overview of laboratory-based syphilis serological tests

\begin{tabular}{|c|c|c|}
\hline Factor & $\begin{array}{c}\text { Specific treponemal } \\
\text { tests }\end{array}$ & $\begin{array}{l}\text { Non-treponemal } \\
\text { tests }\end{array}$ \\
\hline $\begin{array}{l}\text { Types of } \\
\text { laboratory assays }\end{array}$ & $\begin{array}{l}\text { Treponema pallidum } \\
\text { haemagglutination assay } \\
\text { (TPHA) } \\
\text { Treponema pallidum } \\
\text { particle agglutination } \\
\text { assay (TPPA) } \\
\text { Fluorescent treponemal } \\
\text { antibody absorption test } \\
\text { (FTA-Abs) } \\
\text { Treponema pallidum } \\
\text { chemiluminescent } \\
\text { immunoassay (CLIA) } \\
\text { Treponema pallidum } \\
\text { enzyme immunoassay } \\
\text { (EIA) }\end{array}$ & $\begin{array}{l}\text { Rapid plasma reagin } \\
\text { (RPR) } \\
\text { Venereal disease } \\
\text { research laboratory } \\
\text { (VDRL) }\end{array}$ \\
\hline $\begin{array}{l}\text { Performance } \\
\text { characteristics } \\
\text { (sensitivity) }\end{array}$ & $\begin{array}{l}70-90 \% \text { in primary } \\
\text { syphilis } \\
90-100 \% \text { in latent } \\
\text { syphilis }\end{array}$ & $\begin{array}{l}70-80 \% \text { in primary } \\
\text { syphilis } \\
70-100 \% \text { in latent } \\
\text { syphilis }\end{array}$ \\
\hline $\begin{array}{l}\text { Become negative } \\
\text { after treatment? }\end{array}$ & No & $\begin{array}{l}\text { Yes (up to } 25 \% \text { may } \\
\text { remain serofast with } \\
\text { low titres) }\end{array}$ \\
\hline $\begin{array}{l}\text { Measure disease } \\
\text { activity? }\end{array}$ & No & $\begin{array}{l}\text { Yes (expect fourfold } \\
\text { decline in titre in } 6 \\
\text { months to } 1 \text { year } \\
\text { after successful } \\
\text { treatment) }\end{array}$ \\
\hline $\begin{array}{l}\text { False positive } \\
\text { results in } \\
\text { pregnancy? }\end{array}$ & Extremely rare & Can occur \\
\hline
\end{tabular}

lower than with serum, when laboratory-based specific treponemal tests were used as the reference standard. ${ }^{6}$ Nineteen of the 33 studies (58\%) evaluated POCT using whole blood. The specimens came from a variety of sources including sexually transmitted disease clinic attendees; female sex workers; antenatal clinic attendees; and the blood bank. The reference treponemal assays used as comparators, such as TPHA, TPPA and FTA-Abs, were estimated to have sensitivities ranging from $85 \%$ to $100 \%$ and specificities in the range of $98-100 \%$. Under the assumption of an imperfect reference standard, sensitivity estimates with 95\% 'credible' intervals (Cls) for each POCT with whole blood were as follows: Determine Syphilis $86.3 \%$ (77.3, 91.7); SD Bioline 87.1\% (75.7, 94.5); Syphicheck 74.5\% (56.9, 88.4); Visitect $85.1 \%(72.8,92.6)$.

A prospective multi-centre evaluation of four rapid tests (Determine Syphilis, VisiTect Syphilis, SyphiCheck WB, SD Bioline) was conducted at clinics in Tanzania, Brazil, China and Haiti among populations at moderate to high risk of syphilis. ${ }^{7}$ The results revealed a specificity of $>95 \%$ at each site. However, the sensitivities varied by specimen type and place of testing; they were consistently lower for each POCT when performed using whole blood at clinics (64-100\%), compared with use of serum in a laboratory setting (67.4-100\%).

The utility of Determine Syphilis POCT was evaluated in Peruvian commercial sex worker venues in comparison with serum nontreponemal and treponemal assays such as RPR and TPHA, respectively. ${ }^{8}$ The sensitivity of POCT with fingerprick capillary blood was low (39.3\%), but increased with the use of specimens that had corresponding serum RPR titres indicative of active syphilis (64\% with serum RPR $\geq 8$ and $70 \%$ with serum RPR $\geq 16$ ). The sensitivity of POCT using TPHA-positive serum specimens was $97 \%$. It was concluded that the test was

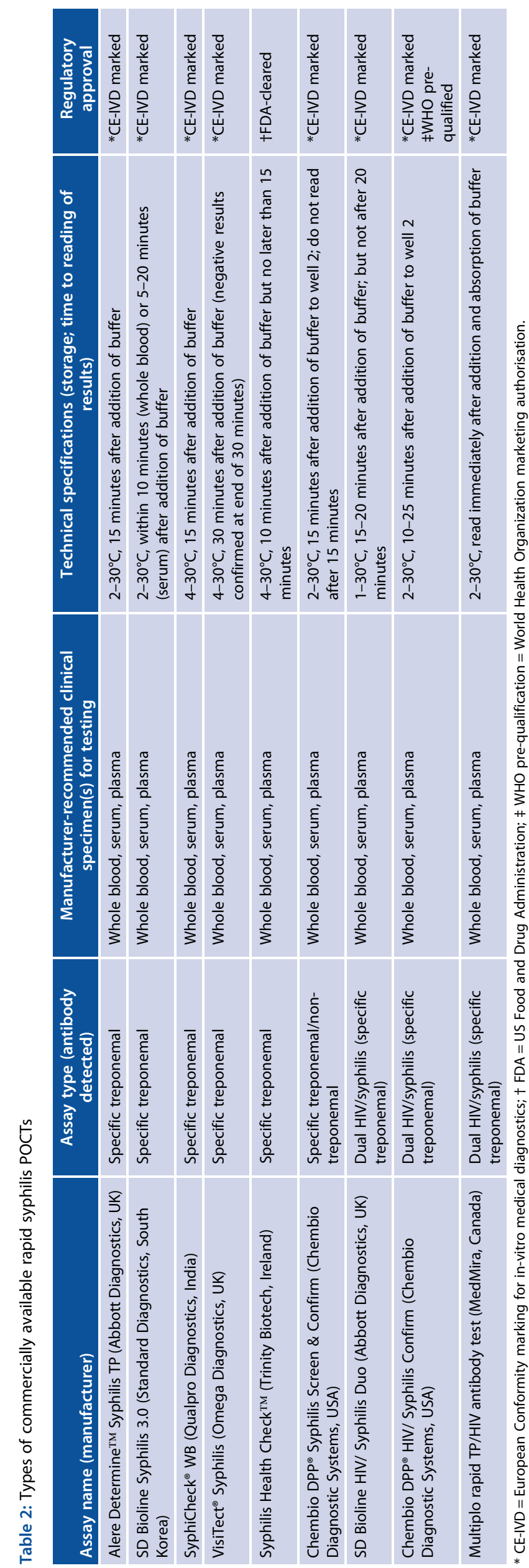


easy to perform and could be integrated into outreach services for sex workers. However, its utility would be low in settings where a large proportion of the population had been previously tested and treated as it detected only specific treponemal antibodies that would be present even after successfully treated syphilis.

In Bengaluru, India, use of SyphiCheck WB among female sex workers was evaluated in comparison with traditional RPR and TPHA laboratory assays. ${ }^{9}$ The sensitivity of the POCT was $70.8 \%$ with whole blood and $97.5 \%$ with serum. This increased to $73.3 \%$ and $100 \%$ for whole blood and serum respectively, when high-titre RPR samples $(\geq 8)$ were used. Due to low rate of patient return for follow-up and treatment, a significantly greater proportion of women with active syphilis were treated using the POCT protocol compared with those that had been managed with standard laboratory testing in the same population during the preceding year $(68.3 \%$ vs. $44.8 \%, p=0.003)$.

Field evaluation of the SD Bioline HIV/Syphilis test among female sex workers accessing primary healthcare and outreach services in inner-city Johannesburg revealed a sensitivity of $66.7 \%$ for syphilis testing using fingerprick blood. ${ }^{10}$ The sample size of patients with active syphilis (RPR $>8$ ) was too small (7/51; $2.8 \%$ ) to accurately determine assay sensitivity for active syphilis; however, it was concluded that the test would be most useful in settings with suboptimal laboratory facilities and support.

\section{Antenatal clinic evaluations}

Evaluation of POCTs in the field is essential for assessing their implementation in 'real-world' situations, where staffing and resource constraints may contribute to suboptimal performance. The utility of Determine Syphilis POCT was assessed in 16 rural antenatal clinics in the Eastern Cape from December 2001 to March 2002; eight clinics that used the on-site POCT intervention were compared with another eight clinics that used standard off-site laboratory screening with serum RPR/ TPHA. ${ }^{11}$ Prevalence of active syphilis was determined to be $6.3 \%$. Six-week interim analysis revealed that on-site POCT had poor sensitivity for detection low-titre syphilis $(\mathrm{RPR}<8)$ due to insufficient capillary blood withdrawn from a fingerprick. Sensitivity improved from $31.3 \%(95 \% \mathrm{Cl} 11-59)$ to $85.7 \%(95 \% \mathrm{Cl} 57-98)$ after the introduction of automatic lancets and calibrated, heparinised capillary tubes. At the intervention clinics compared with standard practice clinics, which experienced low client return rates, a higher proportion of infected pregnant women received at least one dose of intramuscular benzathine penicillin $(89.4 \%$ vs. $60.8 \%)$.

The impact of syphilis POCT was assessed from September 2011 to March 2013 at 18 antenatal facilities in a rural district of Zambia, where the baseline syphilis seroprevalence by RPR screening was estimated to be $2.7 \% .{ }^{12}$ Although the testing coverage improved from $10.3 \%$ to $67.5 \%$ in the first six months of POCT implementation, it had declined to $56.3 \%(p<0.001)$ at the seven-month to one-year endline period. The endline period was characterised by frequent stock-outs of rapid tests, high staff turnover and lack of sustained onsite supervision, as well as inadequate documentation of clinical practice. Additionally, POCT had no impact on treatment coverage for syphilis seropositive women, even though drug stock-outs were not experienced during the intervention period.

It is imperative that syphilis POCTs are successfully integrated into existing screening programmes at facility level. This was highlighted by a systematic review of 2379 maternity clinic records from January 2005 to June 2006 at six public clinics in Northern Cape and Gauteng that assessed antenatal testing and treatment coverage for HIV and syphilis with the use of on-site HIV rapid screening assays and off-site laboratory RPR testing, respectively. ${ }^{13}$ It was revealed that women tested for syphilis were almost four times less likely to be screened for HIV infection at the first antenatal visit, most likely due to specimen collection at different locations. There were also delays to treatment initiation for syphilis-infected women and low antiretroviral therapy coverage rates among HIV-infected women. The findings demonstrated a lack of functional integration of HIV and syphilis prevention-of-mother-to-child transmission (PMTCT) programmes. This problem may to some extent be alleviated by the use of accurate rapid dual HIV/syphilis screening assays at antenatal facilities.

\section{Trade-off between accuracy and accessibility of syphilis POCTs}

As the studies referred to above demonstrate, a definite compromise is required between accuracy and accessibility with regard to the use of syphilis POCTs. This was further highlighted by a field evaluation performed at antenatal facilities in a rural district of Northern Tanzania, where prevalence of active syphilis was $2.3 \%{ }^{14}$ The study revealed that the sensitivity of SD Bioline Syphilis with whole blood was $82 \%$ for high RPR titre specimens, compared with $100 \%$ with plasma EIA. However, the POCT would result in a higher proportion of pregnant women with active syphilis receiving appropriate treatment compared with standard testing ( $82 \%$ vs. $16 \%)$.

The WHO advisory on the use of rapid syphilis tests states that countries with functional screening programmes for antenatal and high-risk populations should maintain the existing system and improve as required. ${ }^{3} \mathrm{~A}$ decision to move to syphilis POCT should be based on a careful assessment of accessibility, quality of testing, treatment coverage and efficacy of the existing programme.

Rapid POCT for syphilis is especially useful for remote rural primary healthcare centres, with limited access to laboratory facilities. Having a syphilis result that may be actioned at first antenatal visit is invaluable for providing care at clinics where women typically present late in pregnancy or do not return regularly for results.

\section{Local epidemiology of syphilis}

Prior to widespread implementation of a screening assay, the population prevalence of disease must be taken into consideration as this, in addition to specific performance characteristics of the test, will impact on its positive predictive value. The most recent national antenatal serosurveillance study, conducted in 2015 , estimated an overall antenatal syphilis prevalence of $2.0 \%$ (95\% Cl 1.8-2.2), which ranged from $1.1 \%$ (95\% Cl 0.8-1.5) in Limpopo province to $4.6 \%(95 \% \mathrm{Cl} 3.8-5.6)$ in the Free State. ${ }^{15}$

Syphilis is also a relatively uncommon cause of genital ulcer disease. The National Institute for Communicable Diseases (NICD) GERMS-SA surveillance findings from 2014 to 2016 show that among patients with genital ulcer disease attending primary healthcare facilities, Treponema pallidum spirochetes were detected in only $6.0 \%(22 / 366 ; 95 \% \mathrm{Cl} 4.0-9.0)$ of ulcerderived specimens. ${ }^{16}$ Among females with vaginal discharge syndrome presenting to a community-based primary healthcare facility in Johannesburg 2007-2014, NICD STI surveillance data 
revealed an active syphilis seroprevalence rate (RPR and TPPA positive) of $2.8 \%$, whereas the prevalence of syphilis-exposed females (RPR negative; TPPA positive) was estimated at $11.4 \%$ (200/1 760) (unpublished data). This has implications for the type of screening assay used for syphilis; a specific treponemal assay as the sole diagnostic test is likely to overestimate those presenting with active syphilis by fourfold.

The definition of 'probable active syphilis', used as a reporting measure by the WHO, should include seroreactivity with both non-treponemal and treponemal tests. Neither test used alone is sufficiently sensitive or specific to identify cases of active syphilis. Furthermore, a review of almost 24000 congenital syphilis cases reported to the US Centres for Disease Control between 1991 and 2009 found no convincing evidence of mother-to-child transmission of syphilis from mothers with persistently negative non-treponemal results during the course of their pregnancy and post-partum period. ${ }^{17}$ This strengthens the argument for the use of an accurate point-of-care dual treponemal/non-treponemal assay.

The recently published WHO guidelines on syphilis screening and treatment for pregnant women recommend that in settings such as ours with a relatively low antenatal prevalence of syphilis $(<5 \%)$, a single on-site rapid treponemal test may be used to screen and treat pregnant women. ${ }^{18}$ This is especially applicable to sites with a low coverage of syphilis screening, high loss to follow-up or limited laboratory capacity. In settings with a high prevalence of syphilis ( $\geq 5 \%)$, sequential testing is recommended with an initial on-site rapid treponemal test (and provision of a first dose of treatment if positive), followed by further off-site RPR testing and additional treatment according to stage of syphilis. Ideally, the phased implementation of rapid POCTs for antenatal syphilis screening should be based on a thorough needs assessment at facility level, and an optimal screening algorithm would incorporate the use of a validated rapid assay(s) that detects the presence of both treponemal and non-treponemal antibodies.

\section{Monitoring and evaluation of EMTCT indicators}

It is essential that the 'path to elimination' of congenital syphilis using rapid syphilis screening tests incorporates a system for the monitoring and evaluation of minimum EMTCT impact and process targets. ${ }^{4}$ Estimation of congenital syphilis incidence has been challenging as case notification rates in the public health sector have historically been suboptimal owing to unclear reporting processes and a lack of real-time reporting platforms. The National Institute for Communicable Diseases is in the process of implementing an enhanced notifiable medical conditions (NMC) surveillance system, which includes strengthening clinician-based NMC notifications through the use of alerts, standardised case definitions and real-time reporting facilities. The process targets for syphilis EMTCT should be routinely monitored using data accessed from the District Health Information System (DHIS) of the National Department of Health. Antenatal syphilis screening and treatment coverage indicators must be included as data elements that are collected and reported by all public healthcare facilities to the National Indicator Dataset (NIDS) of DHIS. These measures will enable validation of the EMTCT process and facilitate evaluation of national progress and global comparisons.

\section{Quality assurance issues}

A robust quality assurance programme is critical for drawing attention to deficiencies in supply chain management and staff training. This was emphasised by a study of the integration of simultaneous triple point-of-care prenatal screening for syphilis, hepatitis B and HIV infection in a rural municipality in Guatemala, with low literacy and poor maternal and child health programmes. ${ }^{19}$ Testing uptake was hampered by high staff turnover and stock-outs of test kits. Fear of disclosure of positive results by healthcare workers led to indeterminate 'weakly-reactive' POCT results being reported as negative. Partner notification was limited by gender inequity and economic vulnerability, factors that are applicable to some of our patient populations.

Dried blood spots have been used extensively in external quality assurance (EQA) programmes for HIV rapid tests; similarly, these have been validated for use in proficiency testing schemes (PTS) for laboratory-based syphilis serological assays, e.g. TPPA. ${ }^{20}$ The development of such assays is needed for proficiency testing of rapid syphilis POCTs at clinical facilities. A centralised EQA scheme would be optimal, preferably with the use of panels of dried tube or clarified serum specimens that correspond to a range of antibody levels giving negative to weakly and strongly reactive POCT results.

\section{Cost-effectiveness studies}

A modelling study has determined that the use of rapid POCT in antenatal settings would be highly cost-effective in sub-Saharan Africa. ${ }^{21}$ It demonstrated a substantial reduction in disability adjusted life years averted (i.e. stillbirths, neonatal deaths and cases of congenital syphilis) at a relatively modest impact on budget. For South Africa, taking into consideration factors such as antenatal syphilis seroprevalence, annual number of livebirths, proportion of women with at least one antenatal visit, per capita gross national income and hourly nursing wages, the intervention was calculated to be cost-effective up to a syphilis seroprevalence of $0.008 \%$. In resource-poor settings of sub-Saharan Africa, the use of a dual treponemal/non-treponemal POCT might also be cost-effective in preventing adverse pregnancy outcomes, while substantially reducing the rate of over-treatment of previously exposed, uninfected women. ${ }^{22}$

\section{Programmatic pitfalls with use of syphilis POCTs}

Some of the studies discussed in this review underscore the problems inherent to field implementation of syphilis POCTs. Figure 1 shows the measures that must be in place for fulfilment of the WHO ASSURED criteria for a syphilis POCT.

Prior to widespread implementation of the assay, the performance characteristics of the POCT should be evaluated in the clinic setting, taking into account the seroprevalence of disease in the population to be screened. It can then be determined whether implementation would significantly impact on testing and treatment coverage in comparison with the existing standard of care.

In resource-poor settings with limited laboratory infrastructure and low patient return rates, the sole use of specific treponemal assays may be justified for the screening and treatment of antenatal syphilis. Utility of dual treponemal/non-treponemal screening assays should be evaluated and considered in populations with a high prevalence of previously exposed and treated persons, in whom the use of treponemal-specific POCTs would result in misdiagnosis and unnecessary treatment.

It is important to note that each POCT has specific technical requirements for storage and time to reading of results. These 


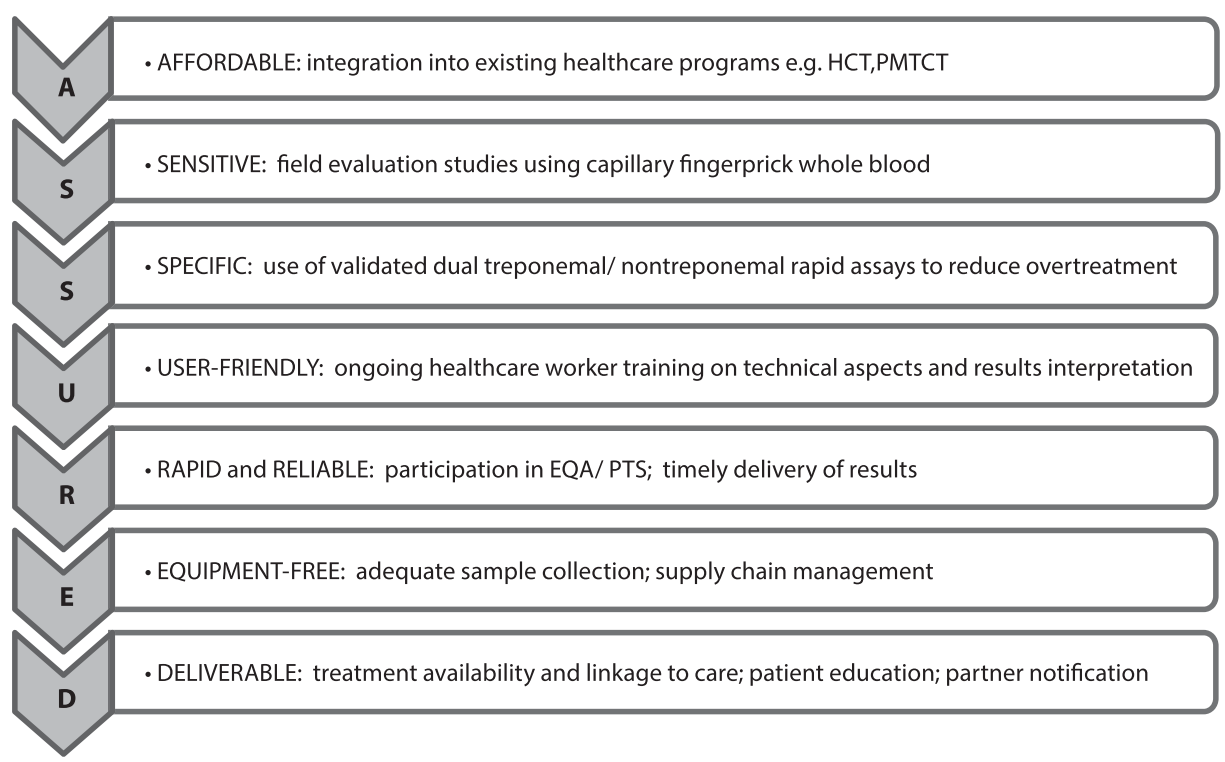

Figure 1: Programmatic requirements for the fulfilment of WHO ASSURED criteria for a syphilis POCT.

criteria should be emphasised when training healthcare workers, especially in facilities with high staff turnover rates. The volume of blood used in testing affects the sensitivity of assay, and use of calibrated capillary tubes may assist in the collection of adequate sample volume. Stock control, stock rotation and supply chain management are essential from a programmatic perspective. Implementation of POCTs in the public health sector should be standardised, so that all healthcare facilities adopting the rapid screening strategy will utilise the same validated assay (s). This will facilitate both user training and bulk procurement.

For the successful programmatic implementation of syphilis POCTs, functional integration into existing sexual and reproductive health programmes is mandatory. The testing should be incorporated into primary healthcare screening and prevention initiatives such as HIV counselling and testing (HCT) for at-risk persons and HIV PMTCT.

External quality assurance is a programmatic requirement for rapid test implementation. Participation in an external proficiency testing scheme should be mandatory, with regular feedback to facilities and investigation, plus corrective action, as necessary.

\section{Future developments}

Next-generation technologies for syphilis POCT will utilise microfluidic sample processing within smaller and more portable devices. These make use of magnetic nanoparticles complexed with antibodies to bind target analyte. The mChip assay developed by scientists at Columbia University, New York, is a plastic dual syphilis/HIV POCT the size of a credit card that uses only 1 microlitre of whole blood. ${ }^{23}$ The electronic reading device is powered by a smartphone through which transmission of results is automated. Increasingly, smartphone-based electronic readers may be used in the field to improve accuracy by removing the element of subjectivity in result reading and interpretation. $^{24}$

\section{Conclusions}

Syphilis POCT implementation in antenatal care is an affordable, feasible and rational intervention for the elimination of congenital syphilis, particularly in resource-constrained settings.
However, widespread implementation in clinic settings requires awareness by policy-makers, programme managers and healthcare workers of the potential programmatic pitfalls, and warrants the institution of measures and operational processes to militate against them.

Disclosure statement - No potential conflict of interest was reported by the author.

\section{References}

1. Wijesooriya NS, Rochat RW, Kamb ML, et al. Global burden of maternal and congenital syphilis in 2008 and 2012: a health systems modelling study. Lancet Glob Health. 2016;4(8):e525-33.

2. Gomez G, Kamb M, Newman L, et al. Untreated maternal syphilis and adverse outcomes of pregnancy: a systematic review and metaanalysis. Bull World Health Organ. 2013;91:217-26.

3. World Health Organisation. The global elimination of congenital syphilis: rationale and strategy for action. 2007. http://www.who. int/reproductivehealth/publications/rtis/9789241595858/en/

4. World Health Organisation. Global guidance on criteria and processes for validation: elimination of mother-to-child transmission of HIV and syphilis. 2014. http://apps.who.int/iris/handle/10665/112858

5. Peeling RW, Holmes KK, Mabey D, et al. Rapid tests for sexually transmitted infections (STIs): the way forward. Sex Transm Infect. 2006;82 (Suppl 5):v1-6.

6. Jafari Y, Peeling RW, Shivkumar S, et al. Are Treponema pallidum specific rapid and point-of-care tests for syphilis accurate enough for screening in resource limited settings? Evidence from a metaanalysis. PLoS One. 2013;8(2):e54695.

7. Mabey D, Peeling RW, Ballard R, et al. Prospective, multi-centre clinicbased evaluation of four rapid diagnostic tests for syphilis. Sex Transm Infect. 2006;82(Suppl 5):v13-6.

8. Campos PE, Buffardi AL, Chiappe M, et al. Utility of the determine syphilis TP rapid test in commercial sex venues in Peru. Sex Transm Infect. 2006;82(Suppl 5):v22-5.

9. Mishra S, Naik B, Venugopal B, et al. Syphilis screening among female sex workers in Bangalore, India: comparison of point-of-care testing and traditional serological approaches. Sex Transm Infect. 2010;86 (3):193-8.

10. Black V, Williams BG, Maseko V, et al. Field evaluation of standard diagnostics' bioline HIV/syphilis duo test among female sex workers in Johannesburg, South Africa. Sex Transm Infect. 2016;92:495-8.

11. Bronzan RN, Mwesigwa-Kayongo DC, Narkunas D, et al. On-site rapid antenatal syphilis screening with an immunochromatographic strip 
improves case detection and treatment in rural South African clinics. Sex Transm Dis. 2007;34(7 Suppl):S55-60.

12. Bonawitz RE, Duncan J, Hammond E, et al. Assessment of the impact of rapid syphilis tests on syphilis screening and treatment of pregnant women in Zambia. Int J Gynaecol Obstet. 2015;130(Suppl 1): S58-62.

13. Dinh TH, Kamb ML, Msimang $V$, et al. Integration of preventing mother-to-child transmission of HIV and syphilis testing and treatment in antenatal care services in the Northern Cape and Gauteng provinces, South Africa. Sex Transm Dis. 2013;40(11):846-51.

14. Smit PW, Mabey D, Changalucha J, et al. The trade-off between accuracy and accessibility of syphilis screening assays. PLoS One. 2013;8 (9):e75327.

15. National Department of Health, South Africa. The 2015 national antenatal sentinel HIV \& syphilis survey, South Africa. 2017. 2015 national antenatal hiv prevalence survey_final_23oct17 (2).pdf

16. Kularatne R. Aetiological surveillance of sexually transmitted infection syndromes at sentinel sites: GERMS-SA 2014-2016. Nat Inst Commun Dis Public Health Surveill Bull S Afr. 2017;15(3):114-22.

17. Peterman TA, Newman DR, Davis $D$, et al. Do women with persistently negative nontreponemal test results transmit syphilis during pregnancy? Sex Transm Dis. 2013;40(4):311-5.

18. World Health Organisation. Who guideline on syphilis screening and treatment for pregnant women. Geneva; 2017. Licence: CC BY-NC-SA
3.0 IGO. file:///F:/EMTCT\%20Syphilis/WHO\%20syphilis\%20screening \%20and\%20treatment\%20pregnancy_2017.pdf

19. Smith A, Sabido M, Camey E, et al. Lessons learned from integrating simultaneous triple point-of-care screening for syphilis, hepatitis $B$, and HIV in prenatal services through rural outreach teams in Guatemala. Int J Gynaecol Obstet. 2015;130(Suppl 1):S70-2.

20. Smit PW, van der Vlis T, Mabey $D$, et al. The development and validation of dried blood spots for external quality assurance of syphilis serology. BMC Infect Dis. 2013;13:S5.

21. Kuznik A, Lamorde M, Nyabigambo A, et al. Antenatal syphilis screening using point-of-care testing in Sub-Saharan African countries: a cost-effectiveness analysis. PLoS Med. 2013;10(11): e1001545.

22. Owusu-Edusei Jr. K, Gift TL, Ballard RC. Cost-effectiveness of a dual non-treponemal/treponemal syphilis point-of-care test to prevent adverse pregnancy outcomes in sub-Saharan Africa. Sex Transm Dis. 2011;38(11):997-1003.

23. Unemo M, Bradshaw CS, Hocking JS, et al. Sexually transmitted infections: challenges ahead. Lancet Infect Dis. 2017;17(8):e235-79.

24. Herbst de Cortina S, Bristow CC, Humphries R, et al. Laboratory evaluation of a smartphone-based electronic reader of rapid dual point-of-care tests for antibodies to human immunodeficiency virus and Treponema pallidum infections. Sex Transm Dis. 2017;44 (7):412-6. 